\title{
Assessment of the risk of contracting Lyme disease in areas with significant human presence
}

[Risco de contrair doença de Lyma em áreas com significativa presença humana]

\author{
M. Milutinović, Ž. Radulović, S. Tomanović \\ University of Belgrade - Institute for Medical Research \\ Dr. Subotića 4, P.O. Box 102 \\ 11129 - Belgrade, Serbia
}

\begin{abstract}
In order to describe seasonal changes in Lyme disease risk rate at three localities in Serbia, a total of 1,542 Ixodes ricinus ticks (493 nymphs; 525 females, and 524 males) were examined from 2003 to 2004. The prevalence of Borrelia burgdorferi in Ixodes ricinus ticks at the Bovan Lake County was higher than the average for European localities $(45.9 \%$ for adults and $18.8 \%$ for nymphs). In Mt. Avala and Kljajićevo counties, adults and nymphs were infected at the following percentages: 26.3 and $10.7 ; 16.2$ and $7.6 \%$, respectively. These outcomes indicate a relatively high risk of the contracting Lyme disease in all investigated areas.
\end{abstract}

Keywords: tick, Ixodes ricinus, Lyme disease, risk rate

\section{RESUMO}

Para estimar a variação sazonal das taxas de risco para doença de Lyme em três localidades da Sérvia foram examinados, no periodo de 2003-2005, 1542 espécimes do carrapato Ixodes ricinus (493 ninfas, 525 fêmeas e 524 machos). A prevalência de Borrelia burgdorferi em Ixodes ricinus no município de Bovan Lake foi mais alta que a registrada em outras localidades da Europa. Nos municipios de Mt. Avala e Kljajićevo as porcentagens de adultos e ninfas infectadas foram: 26,3 e 10,7; 16,2 e 7,6, respectivamente. Esses resultados indicam um relativo alto risco de se contrair doença de Lyme nas três localidades estudadas.

Palavras-chave: carrapato, Ixodes ricinus, doença de Lyme, taxa de risco

\section{INTRODUCTION}

Lyme disease is a multi-system tick-borne disorder caused by the spirochete bacteria Borrelia burgdorferi sensu lato (sl). The most prominent manifestations affect thin skin, nervous system, musculoskeletal system, and heart. Early Lyme disease can cause nonspecific symptoms and diagnosis is often established in late, chronic stages. Assessment of the risk of contracting Lyme disease is an essential component of the design and implementation of treatment and prevention measures.

As the only natural way, humans can been infected with $B$. burgdorferi $s l$ by a tick bite. Predicting the risk is based on the presence of infected ixodid

Recebido em 24 de outubro de 2006 Aceito em 26 de novembro de 2007 E-mail: majam@imi.bg.ac.yu ticks. Risk maps for Lyme disease have been produced by various methods: by detecting the prevalence of borreliae in ixodid ticks (Daniels et al., 1998), by determination of habitat suitability for ixodid tick development (Eisen et al., 2004), or using a geographic information system (Glass et al., 1995; Guerra et al., 2002). The development of new less expensive methods or models for risk assessment is of great interest. Detection of genetic differences between borreliae-infected and uninfected Ixodes ricinus ticks (Radulović, 2005) point to the real possibility of using some genetic markers as indicators of Lyme disease risk.

I. ricinus is the principal vector of Lyme disease in Europe. As B. burgdorferi sl spirochetes are present in all examined $I$. ricinus populations (Hubálek and Halouzka, 1998), the risk of 
contracting Lyme disease exists throughout the geographic distribution of this species, including Europe, Northern Africa, and parts of Asia. There are wide temporal and spatial fluctuations in the risk rate, depending on the abundance of questing $I$. ricinus ticks, the prevalence of borreliae in $I$. ricinus ticks, the intensity of infection of ticks with borreliae, and the gender and stage ratio in tick populations. The importance of these values for Lyme disease risk assessment has alredy been reported (Hubálek et al., 1991, 1994, 2004; Matuschka et al., 1992; Jensen and Frandsen, 2000; Jensen et al., 2000; Nahimana et al., 2004; Cisak et al., 2005). Researches carried out on Lyme disease in Serbia so far pointed out to the significant prevalence of borreliae in $I$. ricinus ticks (Milutinović, 2000; Milutinović et al., 2004).

In this study, important parameters for assessment of Lyme disease risk were recorded at three spatially distant and ecologically different localities in Serbia, during a three-year period in order to describe seasonal changes in risk rates and compare them among those localities.

\section{MATERIAL AND METHODS}

The study was performed at three localities in Serbia, namely: Kljajićevo, Mt. Avala, and Bovan Lake. Ticks were collected within the sampling areas (one for each locality) reaching diameters of $200 \mathrm{~m}$. While the sampling area at the Kljajićevo locality, which is situated below the Telečka loess plateau on the Pannonian plain, consists of agricultural land and sparse deciduous forest (including part of an area used for pheasant breeding), the Mt. Avala and Bovan Lake localities represent recreational areas in the vicinity of Belgrade and Niš, respectively. There is a significant occupational human exposure to tick bites within the studied areas. During the period of questing activity of ticks (from March to November), farmers, hunters, and gamekeepers are present at the Kljajićevo locality, forestry workers at the Mt Avala locality, and anglers and shore guards at the Bovan Lake locality. As for the Mt. Avala and Bovan Lake localities, attendance of a huge number of picnickers during holidays and summer vacations is usual. It should be noted that the waters of Sakinac (included in the study area), a spring situated below Mt. Avala, attracts many visitors.
Host-seeking I. ricinus ticks were collected at each locality once a month from March to November in 2003, 2004, and 2005. Sampling was omitted during December, January, and February due to weather conditions unfavorable for tick activity (low temperature and copious precipitation). Collecting of ticks was performed by dragging a white flannel flag $(1 \times 1 \mathrm{~m})$ over vegetation along $30 \mathrm{~m}$ transects. Over every $5 \mathrm{~m}$ of dragging, all ticks found attached were counted, and all nymphs and adults were put in tubes (separated by gender and stage) with damp paper, and transported live to the laboratory. As contact surface of the flag was $1 \mathrm{~m}^{2}$, the area of each transect was $30 \mathrm{~m}^{2}$. Sampling was conducted from 7a.m. to 5p.m. along 10 semirandomised transects (there were not overlapping between transects), giving a total transect area of $300 \mathrm{~m}^{2}$. Tick abundance was calculated as the average number of ticks per $100 \mathrm{~m}^{2}$.

I. ricinus ticks were examined under dark-field microscopy for borreliae. Every tick was individually dissected, and their abdominal content was homogenized with $20 \mu \mathrm{l}$ of physiological saline on a slide, squashed with a coverslip $(20 \times 20 \mathrm{~mm})$, and examined at magnification of $400 \times$. All optical fields were inspected for at least $20 \mathrm{~min}$ per tick, and borreliae were counted. The intensity of infection of individual ticks by borreliae was roughly evaluated as low (1-9 borreliae per tick), medium (10-100 borreliae per tick), or high ( $>100$ borreliae per tick) (Hubálek et al., 1991).

\section{RESULTS}

The breaking of diapause in ticks and start of active questing for a host are linked with changes of ecological factors in the habitat. Successions of periods of activity and periods of dormancy in the species I. ricinus are caused by physiological changes that are directly dependent upon temperature and relative atmospheric humidity in the external environment (Table 1).

In tracing of seasonal abundance of host-seeking I. ricinus ticks during the three-year period of study at the Kljajićevo, Mt. Avala and Bovan Lake localities, it was noted that the questing activity of ticks started in early spring, during March and April, and continued until late autumn (Fig. 1 and 2). Regarding abundance within localities, seasonal and annual variations were detected for both adults and nymphs. Generally, ticks were more abundant in spring and less in autumn. 
Table 1. Abiotic and biotic characteristics of the investigated localities, relevant to Ixodes ricinus life cycle

\begin{tabular}{|c|c|c|c|c|c|c|c|c|c|c|}
\hline \multirow{5}{*}{\multicolumn{2}{|c|}{$\begin{array}{l}\text { Coordinates } \\
\text { Altitude (meters) } \\
\text { Climate } \\
\end{array}$}} & \multicolumn{9}{|c|}{ Localities } \\
\hline & & \multicolumn{3}{|c|}{ Kljajićevo } & \multicolumn{3}{|c|}{ Mt. Avala } & \multicolumn{3}{|c|}{ Bovan Lake } \\
\hline & & \multicolumn{3}{|c|}{$45^{\circ} 46^{\prime} \mathrm{N} \quad 19^{\circ} 16^{\prime} \mathrm{E}$} & \multicolumn{3}{|c|}{$44^{\circ} 41^{\prime} \mathrm{N} \quad 20^{\circ} 30^{\prime} \mathrm{E}$} & \multicolumn{3}{|c|}{$43^{\circ} 39^{\prime} \mathrm{N} \quad 21^{\circ} 44^{\prime} \mathrm{E}$} \\
\hline & & \multicolumn{3}{|c|}{90} & \multicolumn{3}{|c|}{395} & \multicolumn{3}{|c|}{315} \\
\hline & & & ontinental & & Temp & rate-contin & ntal & Tempe & ate-conti & atal \\
\hline Year & Month & $\mathrm{T}\left({ }^{\circ} \mathrm{C}\right)$ & RH (\%) & $\mathrm{P}(\mathrm{mm})$ & $\mathrm{T}\left({ }^{\circ} \mathrm{C}\right)$ & RH (\%) & $\mathrm{P}(\mathrm{mm})$ & $\mathrm{T}\left({ }^{\circ} \mathrm{C}\right)$ & RH (\%) & $\mathrm{P}(\mathrm{mm})$ \\
\hline \multirow{9}{*}{2003} & III & 5.7 & 67 & 3.0 & 7.1 & 58 & 11.4 & 5.3 & 64 & 5.3 \\
\hline & IV & 11.1 & 57 & 7.8 & 12.1 & 56 & 23.1 & 10.3 & 65 & 48.5 \\
\hline & $\mathrm{V}$ & 20.5 & 56 & 45.3 & 21.5 & 56 & 39.5 & 19.6 & 64 & 38.6 \\
\hline & VI & 24.6 & 54 & 23.0 & 25.0 & 53 & 33.4 & 22.8 & 63 & 44.9 \\
\hline & VII & 23.2 & 59 & 44.6 & 23.1 & 63 & 111.8 & 22.0 & 67 & 49.5 \\
\hline & VIII & 24.5 & 54 & 24.3 & 25.6 & 50 & 6.4 & 23.9 & 54 & 3.0 \\
\hline & IX & 16.5 & 63 & 32.9 & 17.8 & 64 & 57.6 & 15.9 & 73 & 86.5 \\
\hline & $\mathrm{X}$ & 9.5 & 77 & 126.6 & 10.8 & 74 & 115.2 & 9.7 & 81 & 108.1 \\
\hline & XI & 7.5 & 84 & 39.5 & 9.2 & 77 & 23.4 & 8.2 & 79 & 31.5 \\
\hline \multirow{9}{*}{2004} & III & 5.5 & 72 & 39.4 & 7.3 & 64 & 18.9 & 6.3 & 69 & 35.6 \\
\hline & IV & 11.4 & 74 & 109.0 & 12.7 & 67 & 71.7 & 12.3 & 68 & 36.8 \\
\hline & V & 14.4 & 65 & 112.4 & 15.4 & 65 & 63.3 & 13.7 & 73 & 78.3 \\
\hline & VI & 18.9 & 68 & 71.6 & 19.9 & 68 & 113.8 & 18.5 & 74 & 70.8 \\
\hline & VII & 21.1 & 63 & 99.0 & 22.5 & 62 & 94.6 & 21.1 & 68 & 67.1 \\
\hline & VIII & 20.4 & 66 & 33.6 & 21.2 & 69 & 89.3 & 19.5 & 75 & 65.5 \\
\hline & IX & 14.8 & 70 & 57.3 & 16.6 & 70 & 45.0 & 15.3 & 75 & 44.2 \\
\hline & $\mathrm{X}$ & 12.4 & 76 & 85.0 & 14.5 & 75 & 26.5 & 13.4 & 79 & 50.7 \\
\hline & XI & 6.0 & 75 & 91.7 & 7.4 & 76 & 129.5 & 5.9 & 83 & 119.5 \\
\hline \multirow{10}{*}{2005} & III & 4.2 & 68 & 44.3 & 5.6 & 68 & 34.1 & 4.1 & 76 & 42.5 \\
\hline & IV & 10.9 & 65 & 48.1 & 12.2 & 61 & 54.4 & 10.8 & 71 & 98.6 \\
\hline & V & 16.6 & 63 & 44.8 & 17.2 & 65 & 47.4 & 15.5 & 77 & 79.2 \\
\hline & VI & 19.7 & 59 & 77.0 & 20.3 & 63 & 95.1 & 18.2 & 73 & 49.9 \\
\hline & VII & 21.4 & 68 & 193.3 & 22.6 & 69 & 91.4 & 21.1 & 76 & 92.1 \\
\hline & VIII & 19.5 & 73 & 153.7 & 20.6 & 75 & 144.6 & 19.6 & 81 & 147.2 \\
\hline & IX & 17.2 & 74 & 70.3 & 18.4 & 75 & 54.1 & 16.9 & 83 & 57.3 \\
\hline & X & 11.3 & 71 & 4.9 & 12.8 & 71 & 28.2 & 11.1 & 79 & 53.1 \\
\hline & XI & 4.7 & 76 & 17.7 & 6.7 & 76 & 22.8 & 5.2 & 83 & 80.9 \\
\hline & & \multicolumn{3}{|c|}{$\begin{array}{c}\text { Sparse deciduous forest and } \\
\text { agriculture }\end{array}$} & \multicolumn{3}{|c|}{ Mixed deciduous forest } & \multicolumn{3}{|c|}{$\begin{array}{l}\text { Deciduous forest and } \\
\text { meadow }\end{array}$} \\
\hline \multirow{3}{*}{\multicolumn{2}{|c|}{$\begin{array}{c}\text { Frequent } \\
\text { herbaceous } \\
\text { Vegetation } \text { species }^{* *}\end{array}$}} & \multirow{3}{*}{\multicolumn{3}{|c|}{$\begin{array}{l}\text { Medicago sativa } \\
\text { Galium mollugo } \\
\text { Agropyron repens } \\
\text { Urtica dioica }\end{array}$}} & \multirow{3}{*}{\multicolumn{3}{|c|}{$\begin{array}{l}\text { Galium mollugo } \\
\text { Carex hirta } \\
\text { Hedera helix }\end{array}$}} & \multirow{3}{*}{\multicolumn{3}{|c|}{$\begin{array}{l}\text { Polygonum aviculare } \\
\text { Hordeum murinum } \\
\text { Polytrichum commune } \\
\text { Holcus lanatus }\end{array}$}} \\
\hline & & & & & & & & & & \\
\hline & & & & & & & & & & \\
\hline \multicolumn{2}{|r|}{$\begin{array}{l}\text { Dominant } \\
\text { woody } \\
\text { species** }\end{array}$} & \multicolumn{3}{|c|}{ Robinia pseudo-acacia } & \multicolumn{3}{|c|}{$\begin{array}{l}\text { Quercus petraea } \\
\text { Quercus cerris } \\
\text { Fagus silvatica } \\
\text { Cornus mas }\end{array}$} & $\begin{array}{r}Q u \\
Q u\end{array}$ & $\begin{array}{l}\text { ercus cer } \\
\text { rcus petrc }\end{array}$ & \\
\hline $\begin{array}{l}\text { Potential ve } \\
\text { of Ixodes } r i \\
\text { (observatio }\end{array}$ & $\begin{array}{l}\text { rtebrate hosts } \\
\text { cinus ticks } \\
\text { ls) }\end{array}$ & $\begin{array}{r}\text { Apode } \\
\text { Micr } \\
\text { Lep } \\
\text { Vu } \\
\text { Capre } \\
\text { Corv } \\
\text { Phasio }\end{array}$ & $\begin{array}{l}\text { mus agra } \\
\text { otus arva } \\
\text { s europe } \\
\text { pes vulpe } \\
\text { lus capre } \\
\text { is frugile } \\
\text { nus colch }\end{array}$ & & $\begin{array}{r}\text { Apod } \\
\text { Apod } \\
\text { Clethri } \\
\text { Eryn } \\
\text { Sci } \\
T u\end{array}$ & $\begin{array}{l}\text { mus flavic } \\
\text { mus sylva } \\
\text { nomys gla } \\
\text { ceus euro } \\
\text { rus vulgat } \\
\text { dus merul }\end{array}$ & $\begin{array}{l}\text { lis } \\
\text { cus } \\
\text { eolus } \\
\text { eus } \\
\text { s }\end{array}$ & $\begin{array}{r}\text { Apod } \\
\text { Arvi } \\
\text { Eryn } \\
\text { Lel } \\
\text { Sci } \\
V l \\
\mathrm{Tu}\end{array}$ & $\begin{array}{l}\text { mus sylva } \\
\text { ola terres } \\
\text { ceus euro } \\
\text { us europe } \\
\text { rrus vulga } \\
\text { lpes vulp } \\
\text { dus meru }\end{array}$ & $\begin{array}{l}\text { icus } \\
\text { is } \\
\text { eus } \\
\text { s } \\
\text { is } \\
\end{array}$ \\
\hline
\end{tabular}




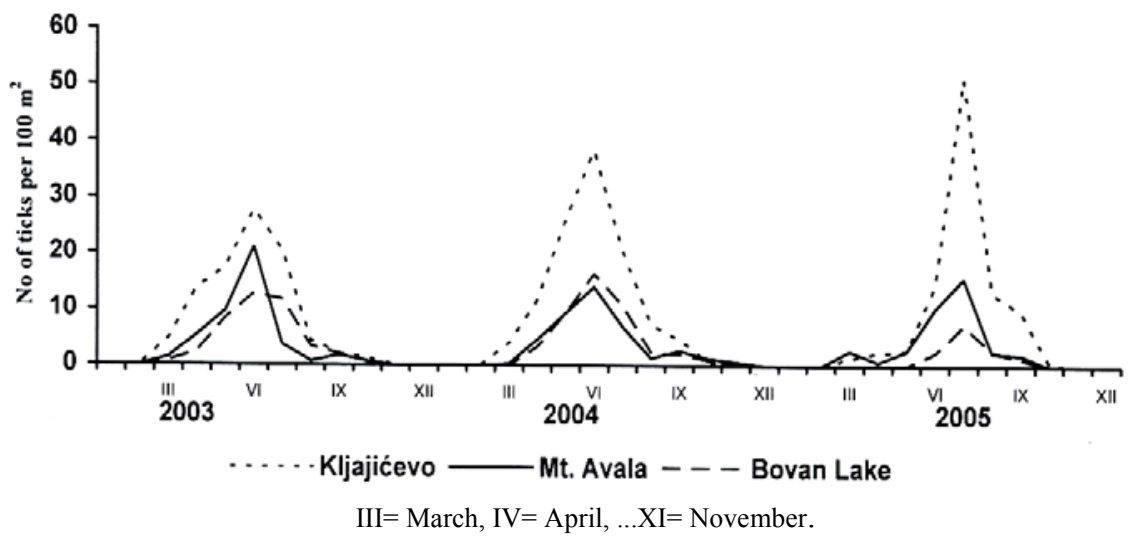

Figure 1. Number of ticks per $100 \mathrm{~m}^{2}$ in three counts in Serbia.

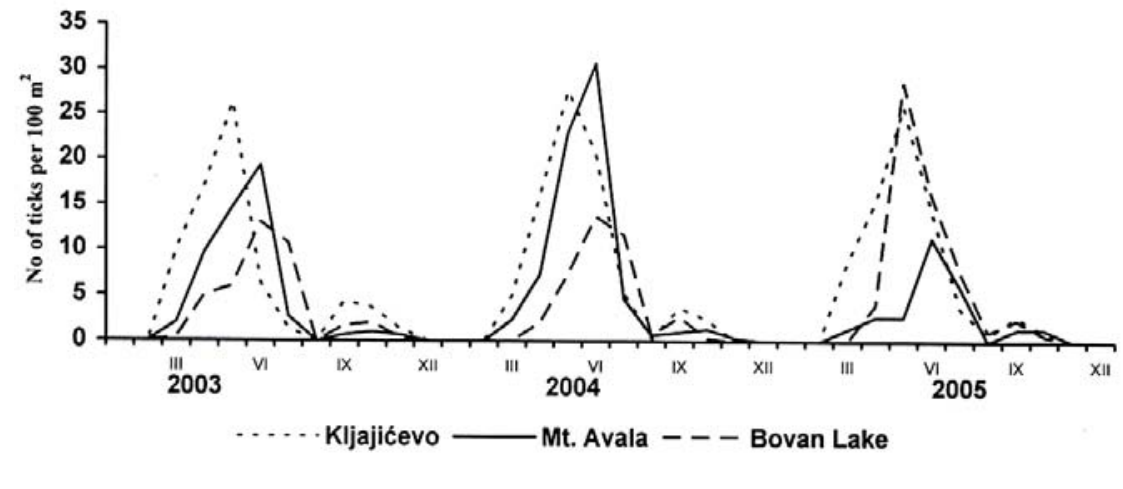

$\mathrm{III}=$ March, $\mathrm{IV}=$ April, ...XI= November.

Figure 2. Seasonal variation of abundance of questing Ixodes ricinus adults in three counts in Serbia.

During the period of 2003-2005, a total of 1,542 I. ricinus ticks (493 nymphs, 525 females, and 524 males) were examined under dark-field microscopy for borreliae presence. Out of this number, $62(12.6 \%)$ nymphs and $308(29.4 \%)$ adults were infected with $B$. burgdorferi sl. The results showed seasonal prevalence of borreliae in questing I. ricinus ticks (Table 2). There were no statistically significant differences of infection rates between males and females at any of the investigated localities. On the other hand, significant differences between adults and nymphs were detected in most annual samples, and differences of total samples for each locality were highly significant (Kljajićevo, $\mathrm{P}=0.008$; $\mathrm{Mt}$. Avala, $\mathrm{P}=0.0001$; Bovan Lake, $\mathrm{P}<0.00001)$. Seasonal and interannual variation in infection rates were insignificant, in contrast to significant differences between localities (for nymphs $\mathrm{P}=0.006$ and for adults $\mathrm{P}<0.00001)$.
As for the approximate intensity of infection, the greatest number of I. ricinus ticks harbored a small number of borreliae, while the proportion of highly infected adults by localities was the following: Kljajićevo - 1.2\%, Mt. Avala - 4.3\%, and Bovan Lake $-12.7 \%$ (Table 3 ). There was only one highly infected nymph, which was collected at the Bovan Lake locality. The seasonal distribution of borreliae infection intensity in nymphs and adults (Table 4) represents information very important for the assessment of the risk of Lyme disease in human and animal populations.

The main reasons for omitting I. ricinus larvae in this study were as follows: low borreliae infection rate (the overall mean prevalence for Europe was about $1.9 \%$ in the review of Hubálek and Halouzka (1998); rare feeding of larvae on humans (Hubálek et al., 2004); and the possibilities of stochastic errors in abundance measuring due to aggregation of larvae after egg eclosion. 


\section{DISCUSSION}

By capturing ticks with the aid of a flag, it is possible to collect only individuals that were actively questing for a host. In addition to active individuals, the total abundance of ticks also included ones that were in diapause and ones in the litter that were making restitution for fluids lost during the active periods. The abundance of active ticks constitutes information more significant than total tick abundance in assessing the degree of risk to the human population, since only active ticks represent a danger to man.

Table 2. Seasonal prevalence of borreliae in Ixodes ricinus ticks in Serbia

\begin{tabular}{|c|c|c|c|c|c|c|c|c|c|c|c|c|}
\hline \multirow{2}{*}{ Area } & \multirow{2}{*}{ Year } & & III & IV & $\mathrm{V}$ & VI & VII & VIII & IX & $\mathrm{X}$ & $\mathrm{XI}$ & Total \\
\hline & & \multicolumn{11}{|c|}{ No. positive/no. examined } \\
\hline \multirow{16}{*}{ 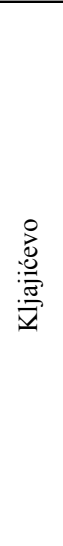 } & \multirow{4}{*}{ હ̊̊ } & Nymph & $1 / 7$ & $1 / 9$ & $1 / 11$ & $0 / 10$ & $1 / 8$ & $1 / 8$ & $0 / 6$ & $1 / 4$ & $0 / 0$ & $6 / 63$ \\
\hline & & Female & $2 / 9$ & $2 / 16$ & $3 / 21$ & $1 / 9$ & $0 / 2$ & $0 / 0$ & $1 / 9$ & $1 / 7$ & $0 / 1$ & $10 / 74$ \\
\hline & & Male & $2 / 13$ & $3 / 13$ & $1 / 10$ & $1 / 10$ & $0 / 2$ & $0 / 0$ & $0 / 4$ & $0 / 4$ & $1 / 3$ & $8 / 59$ \\
\hline & & Adult & $4 / 22$ & $5 / 29$ & $4 / 31$ & $2 / 19$ & $0 / 4$ & $0 / 0$ & $1 / 13$ & $1 / 11$ & $1 / 4$ & $18 / 133$ \\
\hline & \multirow{4}{*}{ 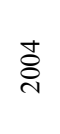 } & Nymph & $0 / 7$ & $1 / 9$ & $0 / 6$ & $2 / 10$ & $1 / 7$ & $0 / 5$ & $0 / 5$ & $0 / 2$ & $0 / 1$ & $4 / 52$ \\
\hline & & Female & $1 / 5$ & $1 / 7$ & $3 / 21$ & $5 / 23$ & $1 / 7$ & $0 / 1$ & $1 / 5$ & $1 / 3$ & $0 / 0$ & $13 / 72$ \\
\hline & & Male & $1 / 6$ & $1 / 6$ & $3 / 18$ & $3 / 20$ & $1 / 9$ & $0 / 0$ & $2 / 7$ & $0 / 3$ & $0 / 0$ & $11 / 69$ \\
\hline & & Adult & $2 / 11$ & $2 / 13$ & $6 / 39$ & $8 / 43$ & $2 / 16$ & $0 / 1$ & $3 / 12$ & $1 / 6$ & $0 / 0$ & $24 / 141$ \\
\hline & \multirow{4}{*}{ 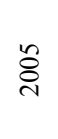 } & Nymph & $0 / 4$ & $0 / 7$ & $1 / 5$ & $0 / 7$ & $0 / 8$ & $0 / 2$ & $1 / 9$ & $0 / 1$ & $0 / 0$ & $2 / 43$ \\
\hline & & Female & $2 / 10$ & $1 / 10$ & $2 / 13$ & $2 / 15$ & $1 / 6$ & $0 / 1$ & $1 / 6$ & $1 / 1$ & $0 / 0$ & $10 / 62$ \\
\hline & & Male & $2 / 12$ & $4 / 12$ & $2 / 12$ & $4 / 19$ & $1 / 5$ & $0 / 1$ & $0 / 2$ & $0 / 2$ & $0 / 0$ & $13 / 65$ \\
\hline & & Adult & $4 / 22$ & $5 / 22$ & $4 / 25$ & $6 / 34$ & $2 / 11$ & $0 / 2$ & $1 / 8$ & $1 / 3$ & $0 / 0$ & $23 / 127$ \\
\hline & \multirow{4}{*}{ 焉 } & Nymph & $1 / 18$ & $2 / 25$ & $2 / 22$ & $2 / 27$ & $2 / 23$ & $1 / 15$ & $1 / 20$ & $1 / 7$ & $0 / 1$ & $12 / 158$ \\
\hline & & Female & $5 / 24$ & $4 / 33$ & $8 / 55$ & $8 / 47$ & $2 / 15$ & $0 / 2$ & $3 / 20$ & $3 / 11$ & $0 / 1$ & $33 / 208$ \\
\hline & & Male & $5 / 31$ & $8 / 31$ & $6 / 40$ & $8 / 49$ & $2 / 16$ & $0 / 1$ & $2 / 13$ & $0 / 9$ & $1 / 3$ & $32 / 193$ \\
\hline & & Adult & $10 / 55$ & $12 / 64$ & $14 / 95$ & $16 / 96$ & $4 / 31$ & $0 / 3$ & $5 / 33$ & $3 / 20$ & $1 / 4$ & $65 / 401$ \\
\hline \multirow{16}{*}{ 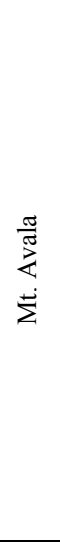 } & \multirow{4}{*}{ §్̊ㅇ } & Nymph & $0 / 4$ & $1 / 11$ & $2 / 13$ & $1 / 14$ & $1 / 7$ & $0 / 2$ & $0 / 5$ & $0 / 2$ & $0 / 0$ & $5 / 58$ \\
\hline & & Female & $1 / 2$ & $2 / 12$ & $2 / 9$ & $6 / 16$ & $1 / 3$ & $0 / 0$ & $0 / 0$ & $0 / 1$ & $0 / 1$ & $12 / 44$ \\
\hline & & Male & $0 / 4$ & $2 / 8$ & $4 / 14$ & $6 / 20$ & $1 / 5$ & $0 / 0$ & $1 / 2$ & $0 / 2$ & $0 / 1$ & $14 / 56$ \\
\hline & & Adult & $1 / 6$ & $4 / 20$ & $6 / 23$ & $12 / 36$ & $2 / 8$ & $0 / 0$ & $1 / 2$ & $0 / 3$ & $0 / 2$ & $26 / 100$ \\
\hline & \multirow{4}{*}{$\underset{ণ}{\stackrel{ণ}{~}}$} & Nymph & $0 / 1$ & $1 / 10$ & $1 / 7$ & $1 / 8$ & $1 / 6$ & $0 / 4$ & $0 / 6$ & $1 / 4$ & $0 / 2$ & $5 / 48$ \\
\hline & & Female & $1 / 1$ & $4 / 9$ & $3 / 13$ & $4 / 13$ & $2 / 8$ & $0 / 0$ & $1 / 1$ & $1 / 1$ & $0 / 0$ & $16 / 46$ \\
\hline & & Male & $1 / 6$ & $4 / 12$ & $1 / 7$ & $3 / 13$ & $1 / 6$ & $1 / 2$ & $0 / 2$ & $1 / 3$ & $0 / 1$ & $12 / 52$ \\
\hline & & Adult & $2 / 7$ & $8 / 21$ & $4 / 20$ & $7 / 26$ & $3 / 14$ & $1 / 2$ & $1 / 3$ & $2 / 4$ & $0 / 1$ & $28 / 98$ \\
\hline & \multirow{4}{*}{ ஜ̊ } & Nymph & $1 / 8$ & $0 / 1$ & $0 / 8$ & $2 / 10$ & $3 / 13$ & $0 / 7$ & $1 / 6$ & $0 / 0$ & $0 / 0$ & $7 / 53$ \\
\hline & & Female & $0 / 1$ & $1 / 3$ & $2 / 4$ & $4 / 17$ & $3 / 9$ & $0 / 0$ & $0 / 3$ & $0 / 3$ & $0 / 0$ & $10 / 40$ \\
\hline & & Male & $1 / 3$ & $2 / 5$ & $1 / 4$ & $2 / 17$ & $2 / 9$ & $0 / 0$ & $0 / 1$ & $1 / 1$ & $0 / 0$ & $9 / 40$ \\
\hline & & Adult & $1 / 4$ & $3 / 8$ & $3 / 8$ & $6 / 34$ & $5 / 18$ & $0 / 0$ & $0 / 4$ & $1 / 4$ & $0 / 0$ & $19 / 80$ \\
\hline & \multirow{4}{*}{ 吾 } & Nymph & $1 / 13$ & $2 / 22$ & $3 / 28$ & $4 / 32$ & $5 / 26$ & $0 / 13$ & $1 / 17$ & $1 / 6$ & $0 / 2$ & $17 / 159$ \\
\hline & & Female & $2 / 4$ & $7 / 24$ & $7 / 26$ & $14 / 46$ & $6 / 20$ & $0 / 0$ & $1 / 4$ & $1 / 5$ & $0 / 1$ & $38 / 130$ \\
\hline & & Male & $2 / 13$ & $8 / 25$ & $6 / 25$ & $11 / 50$ & $4 / 20$ & $1 / 2$ & $1 / 5$ & $2 / 6$ & $0 / 2$ & $35 / 148$ \\
\hline & & Adult & $4 / 17$ & $15 / 49$ & $13 / 51$ & $25 / 96$ & $10 / 40$ & $1 / 2$ & $2 / 9$ & $3 / 11$ & $0 / 3$ & $73 / 278$ \\
\hline \multirow{16}{*}{ 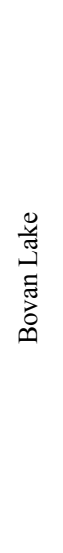 } & \multirow{4}{*}{ 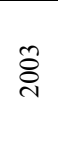 } & Nymph & $0 / 2$ & $0 / 7$ & $3 / 10$ & $5 / 14$ & $4 / 16$ & $1 / 10$ & $1 / 6$ & $0 / 2$ & $0 / 0$ & $14 / 67$ \\
\hline & & Female & $0 / 0$ & $2 / 5$ & $5 / 9$ & $11 / 24$ & $4 / 14$ & $0 / 0$ & $0 / 2$ & $2 / 4$ & $0 / 0$ & $24 / 58$ \\
\hline & & Male & $0 / 1$ & $6 / 10$ & $4 / 9$ & $8 / 15$ & $10 / 18$ & $0 / 0$ & $2 / 3$ & $0 / 2$ & $0 / 1$ & $30 / 59$ \\
\hline & & Adult & $0 / 1$ & $8 / 15$ & $9 / 18$ & $19 / 39$ & $14 / 32$ & $0 / 0$ & $2 / 5$ & $2 / 6$ & $0 / 1$ & $54 / 117$ \\
\hline & \multirow{5}{*}{$\underset{\text { ঠ }}{\stackrel{\overbrace{}}{\circ}}$} & Nymph & $0 / 0$ & $2 / 10$ & $2 / 14$ & $2 / 16$ & $2 / 14$ & $2 / 7$ & $1 / 6$ & $1 / 2$ & $0 / 0$ & $12 / 69$ \\
\hline & & Female & $0 / 0$ & $1 / 1$ & $8 / 14$ & $9 / 17$ & $11 / 17$ & $0 / 1$ & $1 / 3$ & $0 / 1$ & $0 / 0$ & $30 / 54$ \\
\hline & & Male & $0 / 0$ & $3 / 5$ & $4 / 8$ & $4 / 24$ & $9 / 18$ & $1 / 1$ & $3 / 5$ & $0 / 0$ & $0 / 0$ & $24 / 61$ \\
\hline & & Adult & $0 / 0$ & $4 / 6$ & $12 / 22$ & $13 / 41$ & $20 / 35$ & $1 / 2$ & $4 / 8$ & $0 / 1$ & $0 / 0$ & $54 / 115$ \\
\hline & & Nymph & $0 / 0$ & $0 / 0$ & $0 / 0$ & $1 / 7$ & $4 / 22$ & $2 / 7$ & $0 / 4$ & $0 / 0$ & $0 / 0$ & $7 / 40$ \\
\hline & \multirow{3}{*}{ 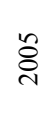 } & Female & $0 / 0$ & $2 / 4$ & $16 / 31$ & $7 / 22$ & $6 / 10$ & $0 / 1$ & $1 / 5$ & $2 / 2$ & $0 / 0$ & $34 / 75$ \\
\hline & & Male & $0 / 0$ & $5 / 8$ & $12 / 23$ & $5 / 15$ & $4 / 13$ & $0 / 2$ & $2 / 2$ & $0 / 0$ & $0 / 0$ & $28 / 63$ \\
\hline & & Adult & $0 / 0$ & $7 / 12$ & $28 / 54$ & $12 / 37$ & $10 / 23$ & $0 / 3$ & $3 / 7$ & $2 / 2$ & $0 / 0$ & $62 / 138$ \\
\hline & \multirow{4}{*}{$\stackrel{\pi}{\stackrel{\pi}{0}}$} & Nymph & $0 / 2$ & $2 / 17$ & $5 / 24$ & $8 / 37$ & $10 / 52$ & $5 / 24$ & $2 / 16$ & $1 / 4$ & $0 / 0$ & $33 / 176$ \\
\hline & & Female & $0 / 0$ & $5 / 10$ & $29 / 54$ & $27 / 63$ & $21 / 41$ & $0 / 2$ & $2 / 10$ & $4 / 7$ & $0 / 0$ & $88 / 187$ \\
\hline & & Male & $0 / 1$ & $14 / 23$ & $20 / 40$ & $17 / 54$ & $23 / 49$ & $1 / 3$ & $7 / 10$ & $0 / 2$ & $0 / 1$ & $82 / 183$ \\
\hline & & Adult & $0 / 1$ & $19 / 33$ & $49 / 94$ & $44 / 117$ & $44 / 90$ & $1 / 5$ & $9 / 20$ & $4 / 9$ & $0 / 1$ & $170 / 370$ \\
\hline
\end{tabular}

$\mathrm{III}=$ March, $\mathrm{IV}=$ April, ...XI $=$ November. 
Milutinović et al.

Table 3. Prevalence of borreliae and approximate intensity of infection in Ixodes ricinus ticks at three locations, 2003-2005

\begin{tabular}{|c|c|c|c|c|c|c|}
\hline \multirow{2}{*}{ Area } & \multirow[b]{2}{*}{ Gender } & \multirow{2}{*}{$\begin{array}{l}\text { No. of } \\
\text { examined }\end{array}$} & \multirow{2}{*}{$\begin{array}{l}\text { Infection } \\
\text { rate }\end{array}$} & \multicolumn{3}{|c|}{ Intensity of infection* } \\
\hline & & & & low & medium & high \\
\hline \multirow{4}{*}{ 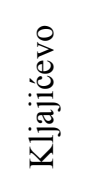 } & & 158 & $7.59 \%$ & $10(6.33 \%)$ & $2(1.27 \%)$ & $0(0.00 \%)$ \\
\hline & \multirow{2}{*}{$\begin{array}{l}\text { Nympl } \\
\text { Adult }\end{array}$} & 401 & $16.21 \%$ & $43(10.72 \%)$ & $17(4.24 \%)$ & $5(1.25 \%)$ \\
\hline & & 193 & $16.58 \%$ & $22(11.40 \%)$ & $6(3.11 \%)$ & $4(2.07 \%)$ \\
\hline & Female & 208 & $15.87 \%$ & $21(10.10 \%)$ & $11(5.29 \%)$ & $1(0.48 \%)$ \\
\hline \multirow{4}{*}{$\begin{array}{l}\frac{\pi}{\pi} \\
\sum^{\pi} \\
\dot{\Sigma} \\
\dot{\Sigma}\end{array}$} & & 159 & $10.69 \%$ & $13(8.18 \%)$ & $4(2.52 \%)$ & $0(0.00 \%)$ \\
\hline & \multirow{2}{*}{$\begin{array}{l}\text { Nympl } \\
\text { Adult }\end{array}$} & 278 & $26.26 \%$ & $30(10.79 \%)$ & $31(11.15 \%)$ & $12(4.32 \%)$ \\
\hline & & 148 & $23.65 \%$ & $14(9.46 \%)$ & $17(11.49 \%)$ & $4(2.70 \%)$ \\
\hline & Female & 130 & $29.23 \%$ & $16(12.31 \%)$ & $14(10.77 \%)$ & $8(6.15 \%)$ \\
\hline \multirow{4}{*}{ 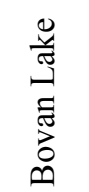 } & & 176 & $18.75 \%$ & $19(10.79 \%)$ & $13(7.39 \%)$ & $1(0.57 \%)$ \\
\hline & \multirow{2}{*}{$\begin{array}{l}\text { Nymph } \\
\text { Adult }\end{array}$} & 370 & $45.95 \%$ & $21(5.68 \%)$ & $102(27.57 \%)$ & $47(12.70 \%)$ \\
\hline & & 183 & $44.81 \%$ & $16(8.74 \%)$ & $44(24.05 \%)$ & $22(12.02 \%)$ \\
\hline & Female & 187 & $47.06 \%$ & $5(2.67 \%)$ & $58(31.02 \%)$ & $25(13.37 \%)$ \\
\hline
\end{tabular}

*Approximate number of borreliae per tick: low, 1-9; medium, 10-100; high, >100.

Table 4. Seasonal distribution of borreliae infection intensity in Ixodes ricinus ticks

\begin{tabular}{|c|c|c|c|c|c|c|c|}
\hline \multirow[b]{2}{*}{ Area } & \multirow[b]{2}{*}{ Month } & \multicolumn{3}{|c|}{ Nymph } & \multicolumn{3}{|c|}{ Adult } \\
\hline & & low & mean & high & low & medium & high \\
\hline \multirow{8}{*}{ Kljajićevo } & \multirow{2}{*}{ III-IV } & $3 / 43$ & & & $17 / 119$ & $4 / 119$ & $1 / 119$ \\
\hline & & $6.98 \%$ & & & $14.29 \%$ & $3.36 \%$ & $0.84 \%$ \\
\hline & \multirow{2}{*}{ V-VI } & $3 / 49$ & $1 / 49$ & & $22 / 191$ & $5 / 191$ & $3 / 191$ \\
\hline & & $6.12 \%$ & $2.04 \%$ & & $11.52 \%$ & $2.62 \%$ & $1.57 \%$ \\
\hline & \multirow{2}{*}{ VII-VIII } & $3 / 38$ & & & $2 / 34$ & $2 / 34$ & \\
\hline & & $7.89 \%$ & & & $5.88 \%$ & $5.88 \%$ & \\
\hline & \multirow{2}{*}{ IX-XI } & $1 / 28$ & $1 / 28$ & & $2 / 57$ & $6 / 57$ & $1 / 57$ \\
\hline & & $3.57 \%$ & $3.57 \%$ & & $3.51 \%$ & $10.53 \%$ & $1.75 \%$ \\
\hline \multirow{8}{*}{ Mt. Avala } & \multirow{2}{*}{ III-IV } & $3 / 35$ & & & $8 / 66$ & $9 / 66$ & $2 / 66$ \\
\hline & & $8.57 \%$ & & & $12.12 \%$ & $13.64 \%$ & $3.03 \%$ \\
\hline & \multirow{2}{*}{ V-VI } & $6 / 60$ & $1 / 60$ & & $14 / 147$ & $16 / 147$ & $8 / 147$ \\
\hline & & $10.00 \%$ & $1.67 \%$ & & $9.52 \%$ & $10.88 \%$ & $5.44 \%$ \\
\hline & \multirow{2}{*}{ VII-VIII } & $3 / 39$ & $2 / 39$ & & $5 / 42$ & $5 / 42$ & $1 / 42$ \\
\hline & & $7.69 \%$ & $5.13 \%$ & & $11.90 \%$ & $11.90 \%$ & $2.38 \%$ \\
\hline & \multirow{2}{*}{ IX-XI } & $1 / 25$ & $1 / 25$ & & $3 / 23$ & $1 / 23$ & $1 / 23$ \\
\hline & & $4.00 \%$ & $4.00 \%$ & & $13.04 \%$ & $4.35 \%$ & $4.35 \%$ \\
\hline \multirow{8}{*}{ Bovan Lake } & \multirow{2}{*}{ III-IV } & $1 / 19$ & $1 / 19$ & & $4 / 34$ & $11 / 34$ & $4 / 34$ \\
\hline & & $5.26 \%$ & $5.26 \%$ & & $11.76 \%$ & $32.35 \%$ & $11.76 \%$ \\
\hline & \multirow{2}{*}{ V-VI } & $7 / 61$ & $5 / 61$ & $1 / 61$ & $7 / 211$ & $63 / 211$ & $23 / 211$ \\
\hline & & $11.48 \%$ & $8.20 \%$ & $1.64 \%$ & $3.32 \%$ & $29.86 \%$ & $10.90 \%$ \\
\hline & \multirow{2}{*}{ VII-VIII } & $9 / 76$ & $6 / 76$ & & $8 / 95$ & $26 / 95$ & $11 / 95$ \\
\hline & & $11.84 \%$ & $7.89 \%$ & & $8.42 \%$ & $27.37 \%$ & $11.58 \%$ \\
\hline & \multirow{2}{*}{ IX-XI } & $2 / 20$ & $1 / 20$ & & $2 / 30$ & $2 / 30$ & $9 / 30$ \\
\hline & & $10.00 \%$ & $5.00 \%$ & & $6.67 \%$ & $6.67 \%$ & $30.00 \%$ \\
\hline
\end{tabular}

$\mathrm{III}=$ March, IV $=$ April, ...XI $=$ November. 
Two peaks were evident in the seasonal abundance of active adults of I. ricinus at the Kljajicevo, Mt. Avala, and Bovan Lake localities; the higher spring peak that occurred during the period of May-June and the lower autumn peak that occurred during the period of September-October, a pattern that is characteristic of populations of this species in the Temperate Zone (Milutinovic, 1992; Milutinovic and Bobic, 1997; Korenberg, 2000; Perret et al., 2000; Milutinovic and Radulovic, 2002). Seasonal dynamics in the abundance of active nymphs of $I$. ricinus at the investigated localities achieved a peak during the period of June-July.

Ecological conditions of the habitat dictate seasonal changes in the number of active ticks, which deviate significantly from the seasonal dynamics of total abundance of the population. Active ticks of the species I. ricinus at the investigated localities were present from April to October, although a significant number of active individuals were also recorded in March at the Kljajicevo and Mt. Avala localities. Only a few active individuals were captured during November of 2004 at the Mt. Avala locality.

The greatest tick abundance was recorded at the Kljajicevo locality, where more than 25 adults per $100 \mathrm{~m}^{2}$ on average were collected during the seasonal peaks. The abundance of adults during the seasonal peaks at the other two localities significantly varied along the years and ranged from about 10 individuals per $100 \mathrm{~m}^{2}$ in June of 2005 to more than 30 individuals per $100 \mathrm{~m}^{2}$ in June of 2004 at the Mt. Avala locality; and from 12 individuals per $100 \mathrm{~m}^{2}$ in June of 2003 to close to 30 individuals per $100 \mathrm{~m}^{2}$ in May of 2005 at the Bovan Lake locality. Interannual variations in the abundance of ticks result from significant interannual differences in the main ecological factors that determine their activity: temperature, atmospheric humidity, and precipitation. The abundance of adults was close to the number recorded by Walker (2001) at a deciduous forest locality in the south of Scotland during the period of 1996-1999, but significantly higher than that recorded in Sweden, namely one adult per $100 \mathrm{~m}^{2}$ on average (Mejlon, 2000). The abundance of nymphs established during the seasonal peaks was lower than expected and 10 times less than the abundance recorded in Wales during the period of 1995-2000 (Randolph et al., 2002). Whereas other authors recorded 10-20 times greater abundance of nymphs in relation to adults at the localities they investigated (Mejlon, 2000; Walker, 2001; Randolph et al., 2002), approximately equal numbers of adults and nymphs on average were captured at the Kljajicevo, Mt. Avala, and Bovan Lake localities. As the main hosts of juvenile stages of I. ricinus, Matuschka et al. (1991) cited small rodents and lizards, which were present in large numbers at the localities they investigated. Nymphs easily found a host and quickly concluded the questing period. Adults prefered to parasitize larger mammals and birds, whose numbers at the investigated localities were small, a circumstance that extended the duration of their questing period. Differences in the duration of active periods were the main factor accounting for capture of a smaller number of nymphs in relation to adults.

The prevalence of $B$. burgdorferi in I. ricinus ticks at the Bovan Lake locality (45.9\% for adults and $18.8 \%$ for nymphs) was exceptionally high and higher than the average for European localities given by Hubalek and Halouzka (1998) (21.1\% for adults and $13.8 \%$ for nymphs). At the Mt. Avala locality, the percentage of infected adults $(26.3 \%)$ was higher, while the percentage of infected nymphs $(10.7 \%)$ was lower than the cited averages. The prevalence of B. burgdorferi in $I$. ricinus ticks at the Kljajicevo locality $(16.2 \%$ for adults and $7.6 \%$ for nymphs) was lower than at the two preceding localities.

Differences between the investigated localities with respect to the number of infected ticks are statistically significant (on the boundary of statistical significance in the case of nymphs) and consistent with the distance between localities. The Mt. Avala locality is approximately midway between the Kljajicevo and Bovan Lake localities. Distance between localities is not a precondition for the existence of differences in the prevalence of borreliae in I. ricinus ticks. Investigating samples of I. ricinus ticks from four neighboring localities on the territory of Belgrade, Milutinovic et al. (2004) detected significant differences between them in the percentage of infected ticks.

In samples of $I$. ricinus ticks from all three of the localities investigated in the present study, the percentage of borrelia-infected nymphs was significantly lower than the percentage of infected adults. Similar results were obtained by Hubalek et al. (1991) at two neighboring localities in the Czech Republic (3.8\% infected nymphs and 10.6\% infected adults at locality A vs. 29.1\% infected 
nymphs and $35.9 \%$ infected adults at locality B). Bukowska et al. (2003) recorded 21.1\% infected adults and $9.9 \%$ infected nymphs during 2000 and 2001 at 10 localities in the immediate vicinity of Szczecin (Poland). At two localities in the south of Sweden, Mejlon (2000) established the presence of 10.1 and $6.9 \%$ infected nymphs and 18 and $19.1 \%$ infected adults of $I$. ricinus, respectively.

Low relative abundance of infected nymphs at the investigated localities does not mean a lower risk of infection after their bites. Due to their small size, nymphs after biting often remain unnoticed by the host for a longer period of time. Sood et al. (1997) indicate a positive correlation between the duration of attachment of an infected tick and infection of the host.

The significance of intensity of tick infection with borreliae for risk assessment is attributable to the shorter period of attachment to the host needed by a highly infected tick in order to successfully transmit the disease agent. In highly infected ticks, transmission of borreliae to the host by saliva during sucking is faster than in the case of weakly infected ticks. The highest prevalence of borreliae in ticks and significantly more highly infected adults were recorded at the Bovan Lake locality in comparison with the other two localities.

In addition to nymphs of $I$. ricinus infected with $B$. burgdorferi, Hubalek et al. (1994) also cite infected females of this species as being potentially dangerous to the human population. Males of $I$. ricinus most often do not feed at all. For assessment of the risk to the human population of infection with borreliae, only the abundance of infected nymphs and females is of any significance.

The abundance of males and females did not significantly differ at the investigated localities, nor did the percentage of their infection in the tested samples. Insignificant variation in the percentage of infected ticks at the investigated localities during 2003, 2004, and 2005, together with the recorded seasonal dynamics in the activity of ticks at these localities, made it possible to define the periods of greatest risk to the human population (Hubalek et al., 1994). In view of the average prevalence and infection intensity recorded at each locality, as well as values of absolute abundance, it is evident that the greatest risk existed at the Bovan Lake locality during May of 2005 , when 14 infected adults per $100 \mathrm{~m}^{2}$ were captured, an average of four of which were highly infected. Despite the significantly greater abundance of ticks (especially nymphs) recorded at the Kljajicevo locality, the risk of contracting Lyme disease is not as high at this locality due to the lower prevalence and small number of highly infected ticks: close to four infected adults per $100 \mathrm{~m}^{2}$, an average of 0.3 of which were highly infected, and about four slightly or moderately infected nymphs during the seasonal peaks of abundance.

Here, it seems appropriate to mention the findings of Radulović (2005) concerning differences in $M d h$ and $\alpha-G p d h$ allele frequencies in $I$. ricinus ticks infected and uninfected with borreliae: The author indicated higher frequencies of rare alleles in infected specimens. Significant differences were confirmed only in females and pertained to the $\alpha-G p d h$ gene locus. These results point to the real possibility of using some genetic markers as indicators of the risk of contracting for Lyme disease. Since approximately the same frequencies of $\alpha-G p d h$ alleles were established at the Kljajicevo, Mt. Avala, and Bovan Lake localities, it would seem logical to expect equal prevalence and intensity of infection with borelliae in these populations of $I$. ricinus. However, the results of the present study contrary to expectations. Many factors affect allele frequencies, and the use of $\alpha-G p d h$ frequency as an indicator of Lyme disease risk must not be completely rejected. Rather, additional investigations are needed to confirm the indicated possibility.

\section{CONCLUSIONS}

The outcomes of this study indicate a relatively high risk of contracting Lyme disease in all investigated areas. The highest risk was recorded at Bovan Lake locality, followed by Mt. Avala and Kljajićevo localities, respectively.

\section{ACKNOWLEDGEMENT}

This work was supported by a grant from the Ministry of Science and Environmental Protection of the Republic of Serbia (Project No. V1726 and V145002) 


\section{REFERENCES}

BUKOWSKA, K.; KOSIK-BOGACKA, D.; KUŹNAGRYGIEL, W. The occurrence of Borrelia burgdorferi sensu lato in the populations of Ixodes ricinus in forest areas of Szczecin during 2000-2001. Ann. Agric. Environ. Med., v.10, p.5-8, 2003.

CISAK, E.; CHMIELEWSKA-BADORA, J.; ZWOLIŃSKI, J. et al. Risk of tick-borne bacterial diseases among workers of Roztocze national park (south-eastern Poland). Ann. Agric. Environ. Med., v.12, p.127-132, 2005.

DANIELS, T.J.; BOCCIA, T. M.; VARDE, S. et al Geographic risk for Lyme disease and human granulocytic ehrlichiosis in southern New York State. Appl. Environ. Microbiol., v.64, p.4663-4669,1998.

EISEN, L.; EISEN, R.J.; CHANG, C.-C. et al. Acarologic risk of exposure to Borrelia burgdorferi spirochaetes: longterm evaluations in north-western California, with implications for Lyme borreliosis risk-assessment models. Med. Vet. Entomol., v.18, p.38-49, 2004.

GLASS, G.E.; SCHWARTZ, B.S.; MORGAN, J.M. III. et al. Environmental risk factors for Lyme disease identified with geographic information systems. Am. J. Public Health., v.85, p.944-948, 1995.

GUERRA, M.; WALKER, E.; JONES, C. et al. Predicting the risk of Lyme disease: Habitat suitability for Ixodes scapularis in the north central United States. Emerging Infect. Dis., v.8, p.289-297, 2002.

HUBÁLEK, Z.; HALOUZKA, J. Prevalence rates of Borrelia burgdorferi sensu lato in host-seeking Ixodes ricinus ticks in Europe. Parasitol. Res., v.84, p.167-172, 1998.

HUBÁLEK, Z.; HALOUZKA, J.; JUŘICOVÁ, Z. A comparison of the occurrence of borreliae in nymphal and adult Ixodes ricinus ticks. Zbl. Bak., v.275, p.133-137, 1991.

HUBÁLEK, Z.; HALOUZKA, J.; JUŘICOVÁ, Z. Borreliae in Ixodes ricinus ticks feeding on humans. Med. Vet. Entomol., v.18, p.228-231, 2004.

HUBÁlEK, Z.; HALOUZKA, J.; JUŘICOVÁ, Z. et al. Seasonal distribution of borreliae in Ixodes ricinus ticks. $Z b l$. Bak., v.280, p.423-431, 1994.

JENSEN, P.M.; FRANDSEN, F. Temporal risk assessment for Lyme borreliosis in Denmark. Scand. J. Infect. Dis., v.32, p.539-544, 2000 .

JENSEN, P.M.; HANSEN, H.; FRANDSEN, F. Spatial risk assessment for Lyme borreliosis in Denmark. Scand. J. Infect. Dis., v.32, p.545-550, 2000.

KORENBERG, E.I. Seasonal population dynamics of Ixodes ticks and tick-borne encephalitis virus. Exp. Appl. Acarol., v.24, p.665-681, 2000.

MATUSCHKA, F.R.; FISCHER, P.; HEILER, M.S. et al. Stage-associated risk of transmission of the Lyme disease spirochete by European Ixodes ticks. Parasitol. Res., v.78, p.695-698, 1992.
MATUSCHKA, F.R.; FISCHER, P.; MUSGRAVE, K. et al. Hosts on which nymphal Ixodes ricinus most abundantly feed. Am. J. Trop. Med. Hyg., v.44, p.100-107, 1991.

MEJLON, H. Host-seeking activity of Ixodes ricinus in relation to the epidemiology of Lyme borreliosis in Sweden . 2000. Dissertation - Faculty of Science and Technology, Uppsala University.

MILUTINOVIĆ, M. Ecological investigations of ticks (Acarina, Ixodoidea, Ixodidae) of Serbia. 1992. Dissertation Faculty of Biology, University of Belgrade, Serbian.

MILUTINOVIĆ, M. Diversity and ecology of the Ixodid ticks (Acari: Ixodidae) in central Serbia, Yugoslavia. Arch. Biol. Sci., v.52, p.39-46, 2000.

MILUTINOVIĆ, M.; BOBIĆ, B. Ecological investigations on ticks (Acari, Ixodidae) of east Serbia, with emphasis on Ixodes ricinus and Hyalomma savignyi. Arq. Bras. Med. Vet. Zootec., v.49, p.531-541,1997.

MILUTINOVIĆ, M.; RADULOVIĆ, Ž. Ecological notes on ticks (Acari: Ixodidae) in Serbia (central regions). Acta Vet., v.52, p.49-58, 2002.

MILUTINOVIĆ, M.; JOVIČIĆ, V. et al. Population dynamics and Borrelia burgdorferi infection rate of Ixodes ricinus ticks in the Belgrade area. Acta Vet., v.54, p.219-225, 2004.

MILUTINOVIĆ, M.; RADULOVIĆ, Ž.; TOMANOVIĆ, S. et al. Seasonal distribution of borreliae in Ixodes ricinus ticks in the Belgrade region, Serbia. Arch. Biol. Sci., v.58, p.183186, 2006.

NAHIMANA, I.; GERN, L.;. BLANCl, D.S. et al. Risk of Borrelia burgdorferi infection in western Switzerland following a tick bite. Eur. J. Clin. Microbiol. Infect. Dis., v.23, p.603-608, 2004.

PERRET, J.L.; GUIGOZ, E.; Rais, O. et al. Influence of saturation deficit and temperature on Ixodes ricinus tick questing activity in a Lyme borreliosis - endemic areas (Switzerland). Parasitol. Res., v.86. p.554-557, 2000.

RADULOVIĆ, Ž. Ecological and genetic study of malate dehydrogenase and a-glycerophosphate dehydrogenase variability in Ixodes ricinus (Linnaeus, 1758) populations. 2005. Thesis - Faculty of Biology, University of Belgrade, Serbian.

RANDOLPH, S.E.; GREEN, R.M.; HOODLESS, A.N. et al. An empirical quantitative framework for the seasonal population dynamics of the tick Ixodes ricinus. Int. J. Parasitol., v.32, p.979-989, 2002.

SOOD, S.K.; SALZMAN, M.B.; JOHNSON, B.J.B. et al. Duration of tick attachment as a predictor of the risk of Lyme disease in an area in which Lyme disease is endemic. $J$. Infect. Dis., v.175, p.996-999, 1997.

WALKER, A.R. Age structure of a population of Ixodes ricinus (Acari: Ixodidae) in relation to its seasonal questing. Bull. Entomol. Res., v.91, p.69-78, 2001. 